\title{
Combined mutation and copy-number variation detection by targeted next-generation sequencing in uveal melanoma
}

Kyra N Smit ${ }^{1,2}$, Natasha M van Poppelen ${ }^{1,2}$, Jolanda Vaarwater ${ }^{1}$, Robert Verdijk ${ }^{3}$, Ronald van Marion ${ }^{3}$, Helen Kalirai ${ }^{4}$, Sarah E Coupland ${ }^{4}$, Sophie Thornton ${ }^{4}$, Neil Farquhar ${ }^{4}$, Hendrikus-Jan Dubbink ${ }^{3}$, Dion Paridaens ${ }^{5}$, Annelies de Klein ${ }^{2}$ and Emine Kiliç ${ }^{1}$

${ }^{1}$ Department of Ophthalmology, Erasmus University Medical Center, Rotterdam, The Netherlands;

${ }^{2}$ Department of Clinical Genetics, Erasmus University Medical Center, Rotterdam, The Netherlands;

${ }^{3}$ Department of Pathology, Erasmus University Medical Center, Rotterdam, The Netherlands; ${ }^{4}$ Department of

Molecular and Clinical Cancer Medicine, University of Liverpool, Liverpool, UK and ${ }^{5}$ The Rotterdam Eye

Hospital, Rotterdam, The Netherlands

\begin{abstract}
Uveal melanoma is a highly aggressive cancer of the eye, in which nearly $50 \%$ of the patients die from metastasis. It is the most common type of primary eye cancer in adults. Chromosome and mutation status have been shown to correlate with the disease-free survival. Loss of chromosome 3 and inactivating mutations in $B A P 1$, which is located on chromosome 3 , are strongly associated with 'high-risk' tumors that metastasize early. Other genes often involved in uveal melanoma are SF3B1 and EIF1AX, which are found to be mutated in intermediate- and low-risk tumors, respectively. To obtain genetic information of all genes in one test, we developed a targeted sequencing method that can detect mutations in uveal melanoma genes and chromosomal anomalies in chromosome 1,3 , and 8 . With as little as $10 \mathrm{ng}$ DNA, we obtained enough coverage on all genes to detect mutations, such as substitutions, deletions, and insertions. These results were validated with Sanger sequencing in 28 samples. In $>90 \%$ of the cases, the BAP1 mutation status corresponded to the BAP1 immunohistochemistry. The results obtained in the lon Torrent single-nucleotide polymorphism assay were confirmed with several other techniques, such as fluorescence in situ hybridization, multiplex ligation-dependent probe amplification, and Illumina SNP array. By validating our assay in $\mathbf{2 7}$ formalin-fixed paraffin-embedded and 43 fresh uveal melanomas, we show that mutations and chromosome status can reliably be obtained using targeted next-generation sequencing. Implementing this technique as a diagnostic pathology application for uveal melanoma will allow prediction of the patients' metastatic risk and potentially assess eligibility for new therapies.

Modern Pathology (2018) 31, 763-771; doi:10.1038/modpathol.2017.187; published online 12 January 2018
\end{abstract}

Uveal melanoma is the most common primary intraocular malignancy in adults with a worldwide annual incidence in Caucasians of 5-7 per million per year. ${ }^{1}$ Despite successful treatment of the primary tumor, nearly $50 \%$ of the patients develop liver metastasis within 5 years. Once metastatic disease is diagnosed, survival is between 2 and 9 months. ${ }^{2}$ Approximately $40 \%$ of uveal melanoma patients developed metastases within 4 years, but

Correspondence: Dr E Kiliç, MD, PhD, Department of Ophthalmology, Erasmus University Medical Center, P.O. Box 2040, Rotterdam 3000 CA, The Netherlands.

E-mail: e.kilic@erasmusmc.nl

Received 21 July 2017; revised 19 October 2017; accepted 19 October 2017; published online 12 January 2018 dissemination can occur even up to 4 decades after diagnosis. ${ }^{3}$ This demonstrates that the prognosis for uveal melanoma patients can strongly vary between patients, and is dependent on a number of factors, including clinical and histological parameters, as well as the underlying genetic 'make up' of the tumor cells. ${ }^{4}$ Chromosomal anomalies are often found in solid tumors, but previous work has shown that most of the chromosomal anomalies in uveal melanoma are limited to chromosome 1, 3, 6, and 8. Some of these chromosomal variations correlated with metastasis, such as loss of chromosome $3 .{ }^{5}$ Monosomy 3 is observed in half of the patients and is strongly associated with poor survival. Loss of chromosome 3 is thought to be an early event, since it is present in the majority of the cells and often accompanies other 
chromosomal anomalies, such as gain of chromosome $8 \mathrm{q} \cdot{ }^{6-8}$ Another common anomaly in metastasizing uveal melanoma with monosomy 3 is loss of chromosome $1 \mathrm{p} .{ }^{9}$ Chromosome 6 shows frequent rearrangements in both p- and q-arm in uveal melanoma; yet, deletion of $6 q$ or gain of $6 p$ are not associated with metastatic disease. ${ }^{10}$ Uveal melanoma are genetically well-characterized tumors. Recent research using genome-wide sequencing led to the discovery of several genetic alterations, which correlate to a distinct survival pattern. Activating mutations in guanine-nucleotide-binding protein-Q (GNAQ) and -alpha 11 (GNA11) were found in the majority of uveal melanoma patients (83-93\%), and are therefore thought to be initiating mutations. ${ }^{11-13}$ Inactivating mutations in the BRCA-associated protein 1 (BAP1), located on chromosome $3 p$, were found in the early metastasizing patients. ${ }^{14}$ Recently, two other genes have been reported that have a role in uveal melanoma biogenesis. Mutations in the eukaryotic translation initiation factor 1A (EIF1AX) were observed in non-metastasizing tumors ${ }^{15}$ and a hotspot mutation in the splicing factor 3 subunit 1 (SF3B1)-gene was detected in late metastasizing tumors. ${ }^{16,17}$ Both of these genes are known to be mutually exclusive.

Current clinical diagnostics for uveal melanoma include several techniques, such as expression profiling, ${ }^{18}$ copy-number analysis by Illumina singlenucleotide polymorphism (SNP)-array, ${ }^{19}$ multiplex ligation-dependent probe amplification ${ }^{20}$ or fluorescence in situ hybridization, ${ }^{21}$ immunohistochemistry of the BAP1 protein, ${ }^{22,23}$ and Sanger sequencing of $E I F 1 A X, S F 3 B 1$, and $B A P 1$. In some cases, wholegenome sequencing or whole-exome sequencing is used to identify the somatic mutations present in the tumor. ${ }^{15,24}$ In this study, we performed Ion Torrent next-generation sequencing with a custom-made panel on 70 uveal melanomas to determine if targeted sequencing can be implemented in the routine uveal melanoma diagnostics. This panel has been designed specifically for uveal melanoma, covering all major hotspot mutations in the five relevant genes and several single-nucleotide polymorphisms on chromosome 1, 3, and 8 to allow analysis of clinically relevant chromosomal anomalies.

\section{Materials and methods}

\section{Uveal Melanoma Samples}

Sixty-five uveal melanoma samples were selected from our Rotterdam Ocular Melanoma Study Groupdatabase and 5 were external samples from patients who underwent enucleation, received for diagnostics from the Liverpool Ocular Oncology Research Group. Samples included in this study were diagnosed as uveal melanoma, collected between 1988 and 2016, and include formalin-fixed paraffinembedded and fresh specimens. A written informed consent was obtained before treatment, the study was performed according to the guidelines of the Declaration of Helsinki, and was approved by the local ethics committee.

\section{DNA Extraction}

Targeted next-generation sequencing was performed on DNA extracted from fresh- and formalin-fixed paraffin-embedded samples. For all tumor samples, an ophthalmic pathologist reviewed and selected tumor areas with an estimated minimal tumor cell percentage of $85 \%$. DNA isolation from fresh tissue was carried out using the QIAmp DNA mini kit (Qiagen, Hilden, Germany), according to the manufacturer's instructions. For formalin-fixed paraffinembedded samples, depending on the size of the tumor, 2-6 $5 \mu \mathrm{m}$ sections were de-paraffinized and hematoxylin-stained prior to isolation of the DNA. Formalin-fixed paraffin-embedded tumor tissue was micro-dissected by scraping the cells manually from hematoxylin-stained sections. DNA was then extracted by incubation of the tissues overnight at $56{ }^{\circ} \mathrm{C}$ in lysis buffer (Promega, Madison, WI, USA), containing 5\% Chelex (Bio-Rad, Berkley, CA, USA) and Proteinase K (Qiagen). Proteinase K was inactivated by incubating the sample for $10 \mathrm{~min}$ at $95^{\circ} \mathrm{C}$ and cell debris was pelleted down together with the Chelex by centrifugation in a microcentrifuge at maximum speed. DNA concentrations were measured with the Quant-iT Picogreen assay kit (Thermofisher Scientific, Grand Island, NY, USA), as described by the manufacturer. All DNA samples were stored at $-20^{\circ} \mathrm{C}$. The DNAs provided by the Liverpool Ocular Oncology Research Group had been extracted as previously described using the Qiagen DNeasy Blood and Tissue kit. ${ }^{25}$

\section{Targeted Next-Generation Sequencing}

A custom primer panel covering the five uveal melanoma genes and several SNPs located on chromosomes 1, 3, and 8, was designed using Ion Ampliseq Designer 2.0 (ThermoFisher Scientific). This resulted in an $11.5 \mathrm{~kb}$ amplicon panel, containing 98 amplicons. Amplicons designed for GNAQ, GNA11, EIF1AX, and SF3B1 covered only the exons containing the known mutation hotspots. All exons of the BAP1 gene were covered by amplicons. On chromosome 1 and 8, 17 amplicons were designed to cover highly polymorphic regions in the entire chromosome (Supplementary Table 1). These highly polymorphic regions with a global minor allele frequency of at least $45 \%$ were selected based on data found in the NCBI SNP database. ${ }^{26}$ For chromosome 3, 21 amplicons were designed, due to the clinical relevance. The DNA input varied between 3 and $10 \mathrm{ng}$, depending on the amount of DNA available per sample. Library construction was performed using the AmpliSeq Library Kit 2.0. 
Next-Generation amplicon sequencing of the libraries was performed by semiconductor sequencing with the Ion Torrent Personal Genome Machine (Thermofisher Scientific) on an Ion Chip, according to the manufacturer's protocol.

\section{Mutation Analysis}

Adapter trimming and filtering of poor quality reads was performed on raw Ion Torrent sequence data by using the platform specific Torrent Suite Software V4.4.3 (Thermofisher Scientific). The generated sequence reads were analyzed with Coverage Analysis and Variant Caller v3.6 plugins to perform sequence coverage analysis and identify variants, respectively. Variants identified as a common polymorphism in the 1000 Genomes-database and variants that were present in $>90 \%$ of the samples were excluded. If variants were present in a frequency higher than $15 \%$ and if they had a minimum read depth of 100 reads, they were called as mutations. Analysis of the detected mutations was done by visualizing the reads in Integrative Genomics Viewer software (Broad Institute, Cambridge, MA, USA) and comparing them to the Ensemble genome database (NM_002072; NM_002067; NM_004656; NM_012433; NM_001412).

\section{Sanger Sequencing}

DNA from 28 tumor samples was sequenced using the Sanger method to confirm results found by nextgeneration sequencing. Selected regions of the genes of interest were amplified by PCR. Subsequently, sequencing of the PCR products and mutation analysis of GNAQ, GNA11, BAP1, and $S F 3 B 1$ and EIF1AX was done as reported previously. ${ }^{13,16,22}$ Alignment of the sequence reads was done with reference sequence Hg19 from the Ensemble genome database.

\section{Immunohistochemical Staining}

To detect loss of the BAP1 protein in tumors, immunohistochemical staining of BAP1 was performed on $4 \mu \mathrm{m}$ formalin-fixed paraffin-embedded sections of tumors. Staining was done by an automated immunohistochemistry staining system (Ventana Medical Systems, Tucson, AZ, USA) as described before. ${ }^{22}$ BAP1 protein expression data were also available for the cases received from Liverpool Ocular Oncology Research Group, which were stained as previously described. ${ }^{27}$ Sections were evaluated by the ophthalmic pathologists in Rotterdam and Liverpool (RV and SEC, respectively).

\section{Copy-Number Variation Analysis}

Validation of the copy-number status of the chromosomes was performed by SNP array, multiplex ligation-dependent probe amplification, and fluorescence in situ hybridization analysis. Two hundred nanograms of fresh tumor DNA was used for the Illuminia 610Q SNP array. Results were analyzed with Nexus Software (BioDiscovery, El Segundo, CA, USA). One hundred nanograms of DNA from each formalin-fixed paraffin-embedded uveal melanoma was used for multiplex ligation-dependent probe amplification analysis of chromosomes 1p, 3, 6 and 8 as previously described. ${ }^{20}$ Fluorescence in situ hybridization analysis was performed on directly fixed tumor material, with probes for chromosome 1 , 3 , and 8 as reported previously. ${ }^{21}$

\section{Results}

\section{Coverage of Uveal Melanoma Genes}

To detect mutations in the GNAQ-, GNA11-, EIF1AX-, SF3B1-, and BAP1 gene, 43 amplicons were used to sequence these genes reliably. Samples with a minimum total read count of 40.000 were analyzed
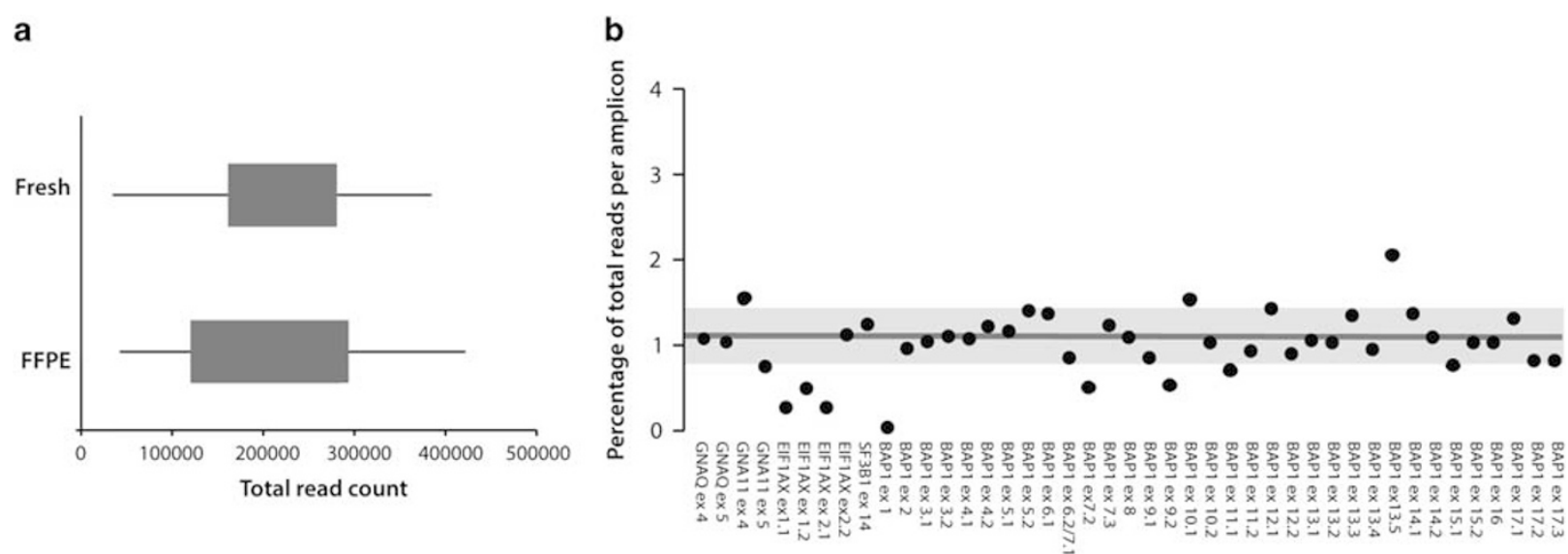

Figure 1 Sequencing efficiency of formalin-fixed, paraffin-embedded, and fresh uveal melanoma specimens. (a) Boxplots showing the total read count for all fresh- (top plot) and formalin-fixed, paraffin-embedded (FFPE) samples (bottom plot). (b) Percentage of total reads visualized for all amplicons covering the five uveal melanoma genes. Solid line indicates median for all amplicons, and light gray area shows second- and third quartile. 
for mutations in the five uveal melanoma genes. The total amount of read counts for fresh samples was on average slightly higher than those of formalin-fixed paraffin-embedded samples (Figure 1a). Most of the amplicons covering the five uveal melanoma genes consisted of $1-2 \%$ of the total read count, which corresponds to a minimum of 400 reads (Figure $1 \mathrm{~b}$ ). The median read count of all amplicons was $1.1 \%$. Several amplicons obtained a coverage of $<1 \%$ of the total read count, such as $E I F 1 A X$ exon 1 and $B A P 1$ exon 1 and 3 . By adding extra amplicons in the primer mix for these areas, we compensated for these lower read counts.

\section{Mutation Analysis}

Seventy uveal melanoma samples were sequenced with our targeted panel. DNA was isolated from fresh specimens $(n=43)$ and from formalin-fixed paraffinembedded material $(n=27)$. From all 70 samples, sufficient DNA was extracted for sequencing. Fortyone percent of the samples harbored a $G N A Q$ exon 5c.626A $>\mathrm{C}$ or c.626A $>\mathrm{T}$ mutation, $3 \%$ a $G N A Q$ exon 4c.548G $>$ A mutation, $41 \%$ a GNA11 exon 5c.626A $>\mathrm{T}$ mutation, $1 \%$ a GNA11 exon 4c.547C $>\mathrm{T}$ mutation, and in the remaining samples no mutations in either of these two genes were detected (Table 1). Mutations in the BAP1 gene were found in $41 \%$ of the cases, mutations in SF3B1 in $16 \%$, and EIF $1 A X$ in $20 \%$ of the samples (Supplementary Table 2). From 28 samples, we extracted enough DNA from fresh tissue to perform Sanger sequencing as well. All the mutations found by next-generation sequencing in these samples were validated by Sanger sequencing and no new mutations were identified.

\section{Detection of Loss of BAP1 Protein Expression}

Absence of the BAP1 protein is often associated with monosomy 3 uveal melanoma. The loss of nuclear BAP1 expression can be immunohistochemically assessed, which is routinely performed in a diagnostic setting. Uveal melanoma samples were sequenced and analyzed for BAP1 mutations. Half of all the samples showed loss of chromosome 3 . Seventy-four percent of these monosomy 3 samples harbored a $B A P 1$ mutation and $26 \%$ did not. BAP1 immunohistochemistry was carried out for 59

Table 1 Amplicon location and mutation rate for the five genes relevant in uveal melanoma

\begin{tabular}{lcccc}
\hline Gene & Chromosome & Exons & Codons & Mutation rate (\%) \\
\hline GNA11 & 19 & 4,5 & 183,209 & 42 \\
GNAQ & 9 & 4,5 & 183,209 & 44 \\
EIF1AX & $\mathrm{X}$ & 1,2 & $4-44$ & 20 \\
SF3B1 & 2 & 14 & 1873,1874 & 16 \\
BAP1 & 3 & $1-17$ & $1-730$ & 41 \\
\hline
\end{tabular}

samples, since we did not have tissue available for immunohistochemistry in all samples. In the $B A P 1-$ mutated samples of which we obtained BAP1 immunohistochemistry data, $80 \%$ showed a negative BAP1 immunohistochemistry $(-), 5 \%$ showed a mixture of positive and negative BAP1 cells in the tumor $(+/-)$, and $15 \%$ showed a positive BAP1 immunohistochemistry (+) (Figure 2 and Supplementary Table 2). The results obtained from three samples are depicted in Figure 3. Hematoxylin and eosin staining indicated a high presence of tumor cells in all three samples (Figure 3a). BAP1 staining was positive for the upper sample and negative for both the middle and lower samples (Figure $3 \mathrm{~b}$ ). Ion Torrent sequencing of the $B A P 1$ gene revealed no mutations in the top sample but did show a mutation in the other two samples (Figure 3c), confirming the presence of BAP1 mutations in the immunohistochemistry BAP1-negative tumors.

\section{Copy-Number Analysis}

SNP array, multiplex ligation-dependent probe amplification, and fluorescence in situ hybridization analyses are commonly used to identify chromosomal changes in tissues. To determine whether the Ion Torrent uveal melanoma custom panel allows a reliable detection of allelic imbalances caused by (partial) losses and gains of chromosome 1, 3 and 8, we compared results obtained by fluorescence in situ hybridization and SNP array with the copy-number variation results from our custom panel. Singlenucleotide polymorphism covering amplicons were evenly distributed over the entire chromosome (Figure 4a), which allowed us to observe partial aberrations as well. Fluorescence in situ hybridization results showed disomy 3 for the top sample and monosomy 3 for the lower sample (Figure $4 \mathrm{~b}$ ). This was confirmed with the SNP array, where the log R Ratio and B-allele frequency shows no loss of heterozygosity for chromosome 3 in the upper sample and monosomy 3 for the lower sample (Figure 4c). The same pattern of allelic distribution was seen with the Ion Torrent single-nucleotide polymorphism analysis of chromosome 3 (Figure 4d). The B-allele frequencies for chromosome 1 and 8 were confirmed as well, as shown in Supplementary Figure 1. Across all samples, we found that $50 \%$ showed monosomy $3,30 \%$ loss of chromosome $1 \mathrm{p}$, and $57 \%$ gain of chromosome $8 \mathrm{q}$. These percentages overlapped with the percentages found by other copy-number variation techniques. Thirty-four samples were validated with only an Illumina SNP array, 15 with SNP array, and fluorescence in situ hybridization, 7 with only fluorescence in situ hybridization, and 5 samples with multiplex ligation-dependent probe amplification (Supplementary Table 3). 


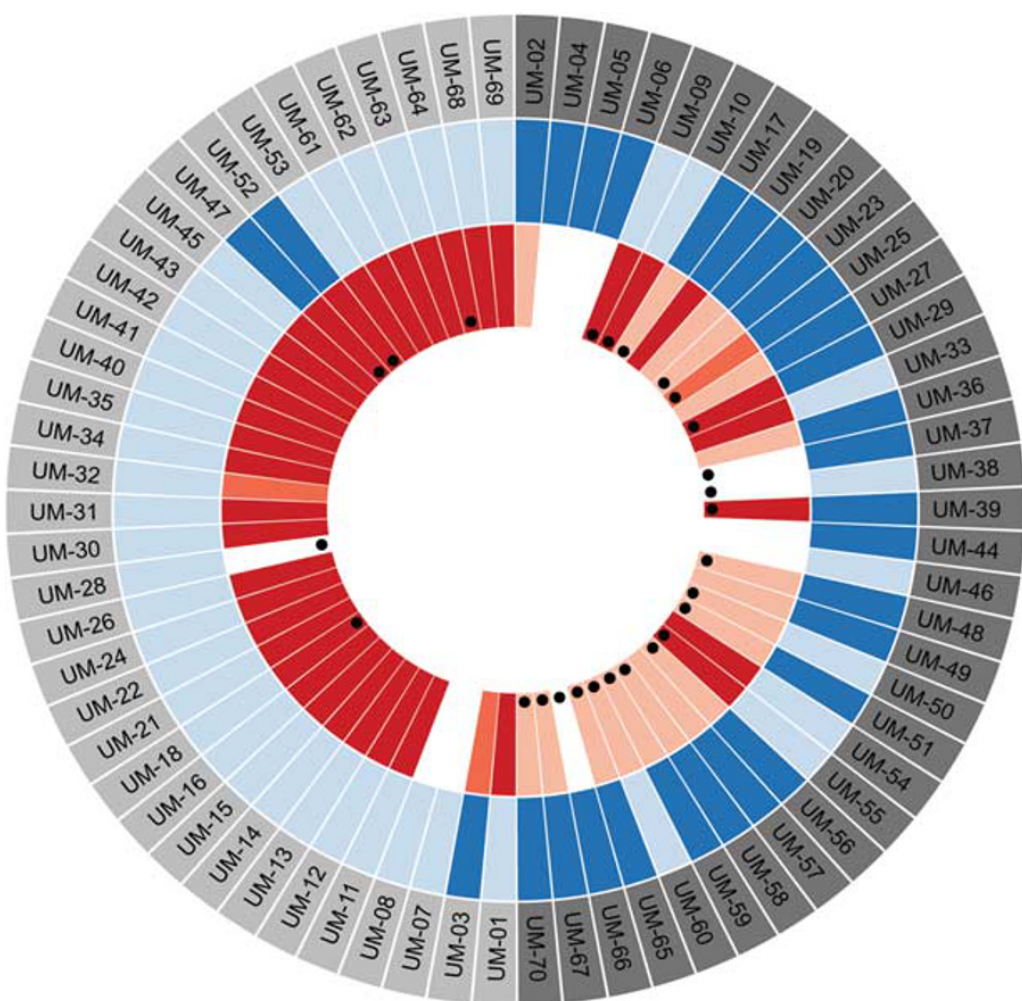

Outer ring: Chromosome 3 status $\square$ Disomy 3

$\square$ Monosomy 3

Middle ring: $B A P 1$ mutation status

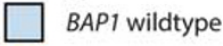

BAP1 mutated

Inner ring: BAP1 IHC

$\square$ Positive BAP1 expression

$\square$ Partial negative BAP1 expression

$\square$ Negative BAP1 expression

$\square$ NE

- Death, due to metastasis

Figure 2 An overview of the overlap between the chromosome 3 status, BAP1 mutation status and BAP1 expression. A doughnut chart visualizing the chromosome 3 status (outer ring), BAP1 mutation status (middle ring), and BAP1 immunohistochemistry (IHC) (inner ring) for all 70 uveal melanoma samples.

\section{Discussion}

Uveal melanoma is characterized by recurrent mutated genes and chromosomal anomalies. In this study, we present a novel custom-designed nextgeneration sequencing assay for uveal melanoma, which can be used to predict uveal melanoma patients' prognoses based on mutation status and chromosome status of chromosome 1,3 , and 8 . The assay can be conducted with using either freshly isolated DNA or DNA obtained from formalin-fixed paraffin-embedded material. This is the first study that establishes a method that can be used for uveal melanoma diagnostics on both formalin-fixed paraffin-embedded and fresh material. Our assay is costeffective, since one method can replace techniques, such as fluorescence in situ hybridization, SNP array, and Sanger sequencing and it can be considered as a good alternative for BAP1 immunohistochemistry. Other important advantages are the low amount of DNA (10 ng) necessary for sequencing, which makes the technique suitable for transvitreal fine needle aspirations biopsies and the small amplicon size, allows sequencing of partially degraded DNA from formalin-fixed paraffinembedded tissue. Our assay could be performed on other next-generation sequencing platforms than Ion Torrent sequencing as well, if these two characteristics are taken into account. Furthermore, compared to other techniques that only identify the high-risk patients that metastasize early, this technique also allows us to identify the potentially late metastasizing patients that often harbor a $S F 3 B 1$ mutation.

Prognostication of uveal melanoma patients can be achieved by analyzing mutation status. Currently, this is usually performed by Sanger sequencing. Mutations in GNAQ, GNA11, and SF3B1, all gain of function mutations, occur almost exclusively in hotspot locations, therefore only these locations have to be sequenced. Since mutations can occur throughout the entire $B A P 1$ gene, large amounts of DNA are needed for the sequencing of multiple exons. Whole-exome sequencing is a reliable and easy method to obtain mutation status as well. However, since only a few genes are involved in the oncogenesis of uveal melanoma, many irrelevant reads will be produced. Whole-exome sequencing is less cost-effective for the diagnostic setting, compared to targeted Ion Torrent sequencing. Several regions of the human genome are difficult to cover with next-generation sequencing. As shown in Figure 1b, a few exons, such as $B A P 1$ exon 1 and the first two exons of EIF1AX, show a relatively low read count. Due to this low read count, it is more difficult to detect mutations in this particular exon. These findings are not only observed in our targeted uveal melanoma panel, but also in whole-genome sequencing data of uveal melanoma. ${ }^{17,28}$ Since exon 1 of the BAP1 gene is located in the non-translated 

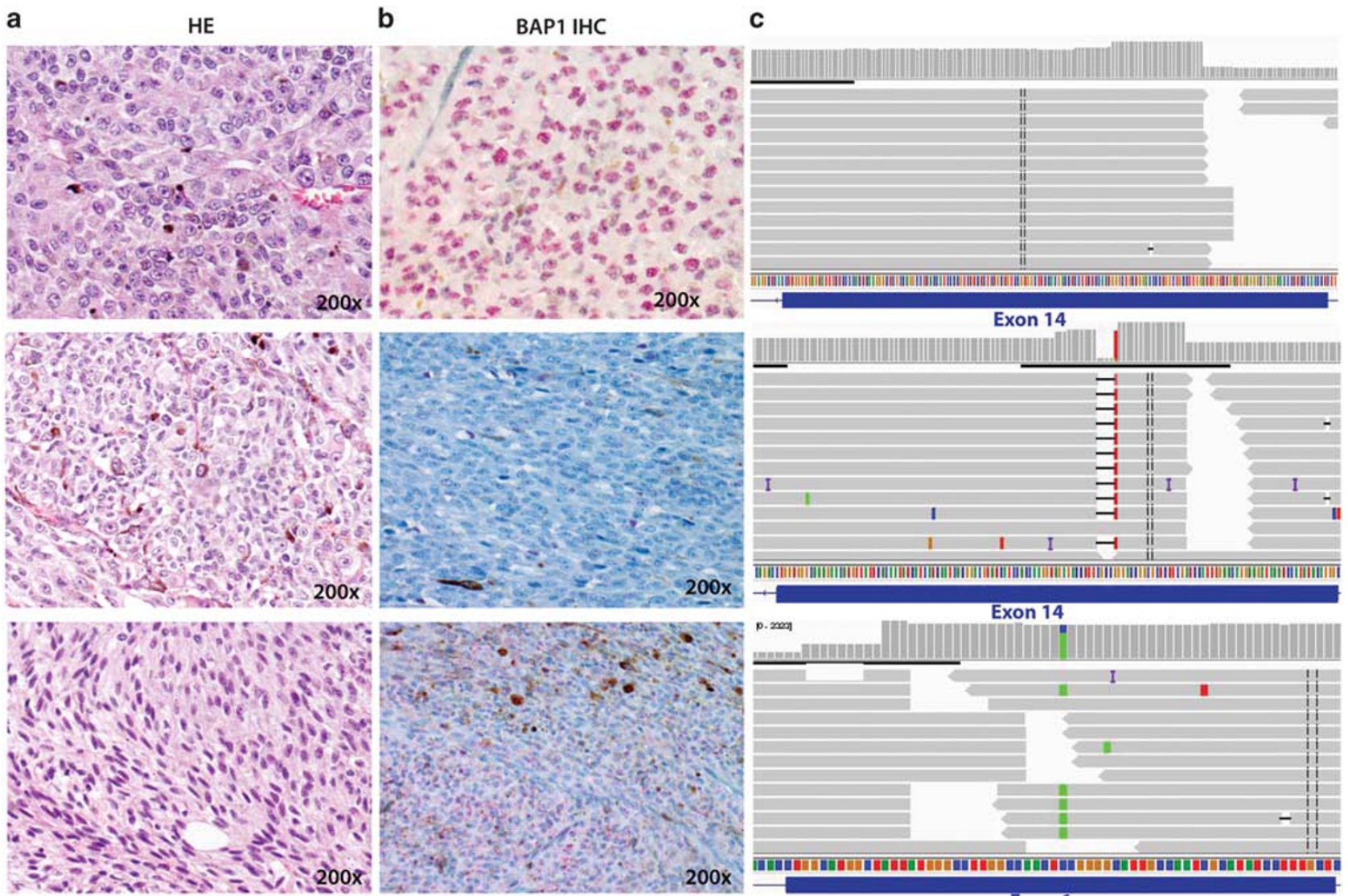

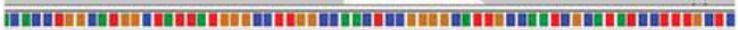

Exon 6

Figure 3 Histopathological and genetic aspects of three uveal melanoma specimens. (a) Hematoxylin and eosin staining (HE) of three uveal melanoma samples $(200 \times)$. (b) Immunohistochemistry (IHC) staining of BAP1 protein showing strong nuclear BAP1 expression in the top sample and loss of BAP1 expression in middle and bottom sample $(200 \times)$. (c) From top to bottom: no mutation observed in the BAP1-gene, a 5 bp deletion and insertion in exon 4 resulting in a frameshift (c.175_179delinsA ) and a point mutation in exon 6 that changes a glutamate into a STOP-codon (c.406G $>$ T).

region, the effect of a mutation in this UTR region is not always clear. Another region, which is sensitive for sequencing errors is exon 1 of EIF1AX, caused by a pseudogene on chromosome 1. Amplicons covering only exon 1 may also produce reads derived from chromosome 1. By adding a second set of reads generated by a different amplicon for $E I F 1 A X$, we now cover not only exon 1 but also a part of the $3^{\prime} \mathrm{UTR}$, which will obtain longer reads that can only be derived from EIF1AX exon 1. In our cohort, we observed mutations in all of the major uveal melanoma genes. Eighty-six percent of the samples showed a mutation in GNAQ or GNA11. Mutations in EIF1AX were found in $20 \%$, mutations in $S F 3 B 1$ in $16 \%$, and mutations in $B A P 1$ were detected in $41 \%$ of the cases. The obtained results do not exactly overlap with the mutation rates for uveal melanoma that we previously reported, ${ }^{16}$ but those differences can be explained by the bias in our sample population. Samples selected for this study were not randomly chosen, but rather selected based on follow-up length and tissue availability. Figure 2 shows that only $74 \%$ of the monosomy samples harbor a BAP1 mutation, which can be explained by studies showing that $B A P 1$ mutations arise after loss of chromosome $3 .{ }^{29}$ Most of the BAP1-mutated samples showed a negative BAP1 immunohistochemistry, but some had positive and negative BAP1 immunohistochemistry cells, which possibly indicates that not all of the cells in the tumor have acquired the mutation yet. However, we also observed BAP1-mutated samples that showed a positive BAP1 immunohistochemistry. For the disomy 3 samples, this can be explained by the presence of a BAP1 wild-type gene, but this is not the case for the monosomy 3 samples. In these samples, we hypothesize that the mutated mRNA is not degraded by nonsense mediated decay and could thereby still be translated into a partially functional or non-functional protein. If the antibody binds at a different location as where the mutation is found, it will show a positive immunohistochemistry. However, for the majority $(91.6 \%)$ of the samples the uveal melanoma panel can correctly detect mutations corresponding to the observed loss of BAP1 expression.

Besides mutation status analysis, our panel also provides information about the copy-number status. Techniques such as fluorescence in situ hybridization, multiplex ligation-dependent probe amplification and SNP array can provide information about the chromosomal change of one or several chromosomes in the tumor in most cases, but these 
a

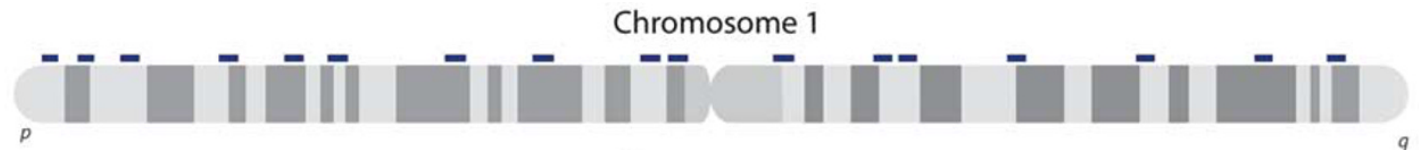

Chromosome 8

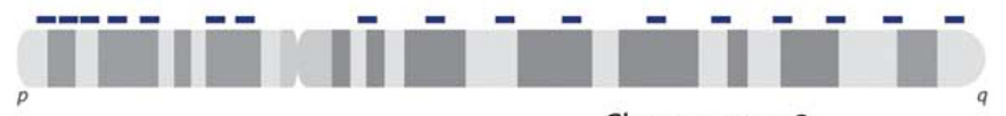

Chromosome 3

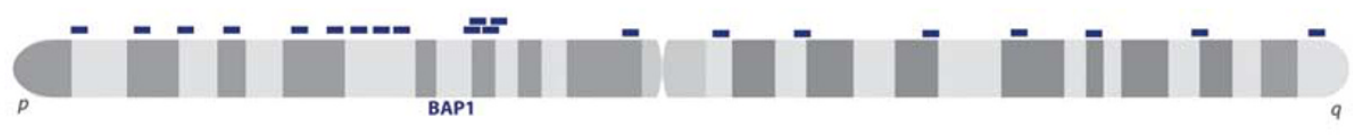

b FISH of chromosome 3
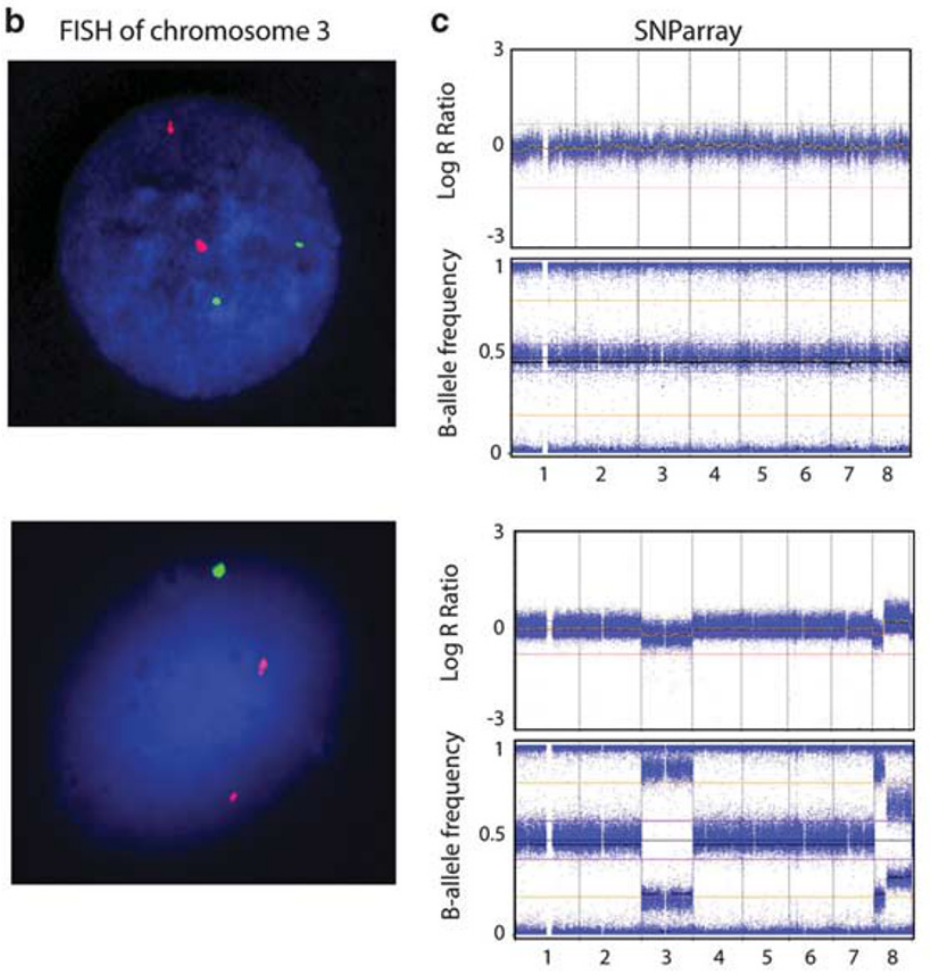

d
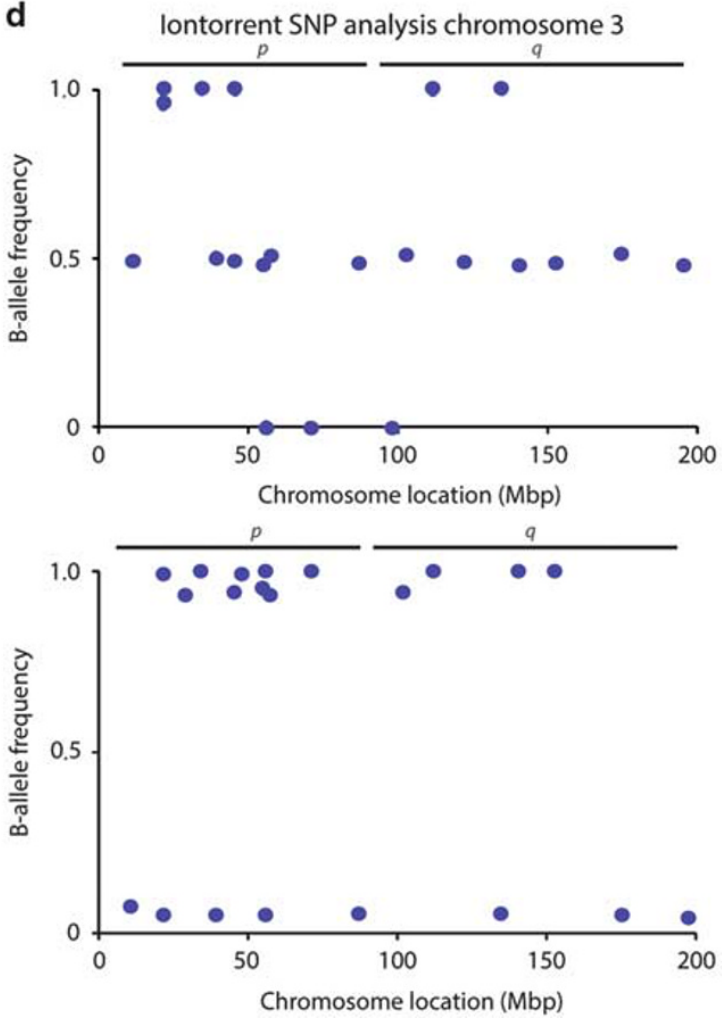

Figure 4 Copy-number analysis of chromosome 3. (a) Visualization of the evenly spread amplicons covering highly polymorphic singlenucleotide polymorphisms on chromosome 1, 3, and 8. (b) Fluorescence in situ hybridization (FISH) of chromosome 5 (red) and chromosome 3 (green) shows no loss for chromosome 3 in the top sample and loss of chromosome 3 in the bottom sample. (c) Top SNP array visualizes chromosome status for chromosome 1-8. Both log R Ratio and B-allele frequency indicate disomy 3 , whereas the SNP array for the bottom panel shows loss of chromosome 3 (d) Single-nucleotide polymorphism (SNP) analysis performed by the targeted uveal melanoma panel visualizes the B-allele frequency for chromosome 3. Top single-nucleotide polymorphism analysis shows heterozygosity for the single-nucleotide polymorphisms, indicating disomy 3, while bottom sample shows no heterozygous variants indicating loss of heterozygosity of chromosome 3.

techniques also have their disadvantages. The probes used for fluorescence in situ hybridization are specific for a certain region, that is, fluorescence in situ hybridization testing does not screen the entire chromosome. It is also a relatively laborious technique, which can take up to several days. Performing a SNP array requires less time, but the amount of DNA necessary (200 ng) is significantly higher than other techniques. Furthermore, standard SNP array is less successful on DNA extracted from formalin-fixed paraffin-embedded tissue compared to freshly obtained DNA. With our uveal melanoma panel, we reliably detect copy-number variations by sequencing of highly polymorphic single-nucleotide polymorphisms. Since this assay requires less DNA than conventional SNP-arrays and less time than fluorescence in situ hybridization, it is a promising method for routine uveal melanoma diagnostics. Copy-number analysis by next-generation sequencing can be challenging in case of low tumor percentages, but since uveal melanoma typically tend to have a high tumor cell content and little heterogeneity for chromosome 3 we do not expect this will pose a problem in our assay. ${ }^{30}$ Chromosome 1 and 8 might have more heterogeneity, thus in case of low tumor cell content and non-conclusive Ion 
Torrent single-nucleotide polymorphisms array results, additional experiments might be necessary. The single-nucleotide polymorphism analysis performed with this uveal melanoma panel does not allow detection of polyploidy in samples. However, recently it has been shown that polyploidy in uveal melanoma does not change the mutation prevalence, which means that detecting polyploidy in uveal melanoma patients has little impact in this method since it does not affect the prognosis. ${ }^{31}$

Our Ion Torrent uveal melanoma panel is in the current state already suitable for implementation in uveal melanoma prognostication, with the advantage that it can easily be expanded by adding the more recently discovered genes into our panel. Recently, it has been reported that a small percentage of the uveal melanoma samples contain mutations in other spliceosome components, SR2F2 and U2AF1. It is thought that these tumors act in the same way as SF3B1-mutated tumors. ${ }^{32}$ Other rare alterations in uveal melanoma are mutations in PCLB4 and CYSTLR2, which are downstream targets of GNA11 and GNAQ and are thereby thought to be less suitable for prognostication. ${ }^{33}$

In summary, we present a next-generation sequencing-based assay that can readily be implemented as a diagnostic pathology application for uveal melanoma. Mutation and copy-number variation data can be obtained by one technique, which can reliably predict the patients' outcome and potentially assess eligibility for new therapies. At present, there is no successful treatment for metastasized uveal melanoma; however, with the development of new therapies, identification of high-risk patients will be very important, particularly in adjuvant therapy trials. Our custom-designed uveal melanoma panel will make a valuable contribution to the rapid stratification of uveal melanoma patients.

\section{Acknowledgments}

We thank Quincy van den Bosch for his assistance with imaging and Tom Brands and Tom de VriesLentsch for helping with the figures. This study was supported by a grant of the Combined Ophthalmic Research Rotterdam.

\section{Disclosure/conflict of interest}

The authors declare no conflict of interest.

\section{References}

1 Singh A, Turell M, Topham A. Uveal melanoma: trends in incidence, treatment and survival. Ophthalmology 2011;118:1881-1885.

2 Woodman S. Metastatic uveal melanoma: biology and emerging treatments. Cancer J 2012;18:148-152.
3 Coupland S, Sidiki S, Clark B. Metastatic choroidal melanoma to the contralateral orbit 40 years after enucleation. Arch Ophthalmol 1996;114:751-756.

4 Damato B, Eleuteri A, Taktak A, et al. Estimating prognosis for survival after treatment of choroidal melanoma. Prog Retin Eye Res 2011;30:285-295.

5 Prescher G, Bornfeld N, Becher R. Nonrandom chromosomal abnormalities in primary uveal melanoma. J Natl Cancer Inst 1990;82:1765-1769.

6 van den Bosch T, van Beek J, Vaarwater J, et al. Higher percentage of FISH-determined monosomy 3 and $8 \mathrm{q}$ amplification in uveal melanoma cells relate to poor patient prognosis. Invest Ophthalmol Vis Sci 2012;15: 2668-2674.

7 de Lange M, van Pelt S, Versluis M, et al. Heterogeneity revealed by integrated genomic analysis uncovers a molecular switch in malignant uveal melanoma. Oncotarget 2015;6:37824-37835.

8 Dogrusöz M, Bagger M, van Duinen S, et al. The prognostic value of AJCC staging in uveal melanoma is enhanced by adding chromosome 3 and $8 \mathrm{q}$ status. Invest Ophthalmol Vis Sci 2017;58:833-842.

9 Kilic E, Naus N, van Gils W, et al. Concurrent loss of chromosome arm $1 p$ and chromosome 3 predicts a decreased disease-free survival in uveal melanoma patients. Invest Ophthalmol Vis Sci 2005;46:2253-2257.

10 White V, Chambers J, Courtright P, et al. Correlation of cytogenetic abnormalities with the outcome of patients with uveal melanoma. Cancer 1998;83:354-359.

11 van Raamsdonk C, Bezrookove V, Green G, et al. Frequent somatic mutations of GNAQ in uveal melanoma and blue naevi. Nature 2009;457:599-602.

12 van Raamsdonk C, Griewank K, Crosby $\mathrm{M}$, et al. Mutations in GNA11 in uveal melanoma. N Engl J Med 2010;363:2191-2199.

13 Koopmans A, Vaarwater J, Paridaens D, et al. Patient survival in uveal melanoma is not affected by oncogenic mutations in GNAQ and GNA11. Br J Cancer 2013;109:493-496.

14 Harbour J, Onken M, Roberson E, et al. Frequent mutations of BAP1 in metastasising uveal melanomas. Science 2006;330:1410-1413.

15 Martin M, Masshöfer L, Temming P, et al. Exosome sequencing identifies recurrent somatic mutations in EIF1AX and SF3B1 in uveal melanoma with disomy 3. Nat Genet 2013;45:933-936.

16 Yavuzyigitoglu S, Koopmans A, Verdijk R, et al. Uveal melanomas with SF3B1 mutations: a distinct subclass associated with late-onset metastases. Ophthalmology 2016;123:1118-1128.

17 Furney S, Pedersen M, Gentien D, et al. SF3B1 mutations are associated with alternative splicing in uveal melanoma. Cancer Discov 2013;3:1122-1129.

18 Onken M, Worley L, Ehlers J, et al. Gene expression profiling in uveal melanoma reveals two molecular classes and predicts metastatic death. Cancer Res 2004;64:7205-7209.

19 Ewens K, Kanetsky P, Richards-Yutz J, et al. Genomic profile of 320 uveal melanoma cases: chromosome 8ploss and metastatic outcome. Invest Ophthalmol Vis Sci 2013;54:5721-5729.

20 Damato B, Dopierala J, Coupland S. Genotypic profiling of 452 choroidal melanomas with multiplex ligationdependent probe amplification. Clin Cancer Res 2010;16:6083-6092.

21 Vaarwater J, van den Bosch $\mathrm{T}$, Mensink $\mathrm{H}$, et al. Multiplex ligation-dependent probe amplification 
equals fluorescence in-situ hybridization for the identification of patients at risk for metastasic disease in uveal melanoma. Melanoma Res 2012;22:30-37.

22 Koopmans A, Verdijk R, Brouwer R, et al. Clinical Significance of immunohistochemistry for detection of BAP1 mutations in uveal melanoma. Mod Pathol 2014;27:1321-1330.

23 van den Nes J, Nelles J, Kreis S, et al. Comparing the prognostic value of BAP1 mutation pattern, chromosome 3 status and BAP1 immunohistochemistry in uveal melanoma. Am J Surg Pathol 2016;40:796-805.

24 Royer-Bertrand B, Torsello M, Rimoldi D, et al. Comprehensive genetic landscape of uveal melanoma by whole-genome sequencing. Am J Hum Genet 2016;99:1190-1198.

25 Lake S, Kalirai H, Dopierala J, et al. Comparison of formalin-fixed and snap-frozen samples analysed by multiplex ligation-dependent probe amplification for prognostic testing in uveal melanoma. Invest Ophthalmol Vis Sci 2012;53:2647-2652.

26 Dubbink HJ, Atmodimedjo P, van Marion $\mathrm{R}$, et al. Diagnostic detection of allelic losses and imbalances by next-generation sequencing $1 \mathrm{p} / 19 \mathrm{q}$ co-deletion analysis of gliomas. J Mol Diagn 2016;18:775-786.
27 Kalirai H, Dodson A, Faqir S, et al. Lack of BAP1 protein expression in uveal melanoma is associated with increased metastatic risk and has utility in routine prognostic testing. Br J Cancer 2014;111: 1373-1380.

28 Johansson P, Aoude L, Wadt K, et al. Deep sequencing of uveal melanoma identifies a recurrent mutation in PLCB4. Oncotarget 2016;7:4624-4631.

29 Robertson A, Shih J, Yau C, et al. Integrative analysis identifies four molecular and clinical subsets in uveal melanoma. Cancer Cell 2017;32:204-220.

30 Mensink HW, Vaarwater J, Kiliç E, et al. Chromosome 3 intratumor heterogeneity in uveal melanoma. Invest Ophthalmol Vis Sci 2009;50:500-504.

31 Yavuzyigitoglu S, Mensink H, Smit K, et al. Metastatic disease in polyploid uveal melanoma is associated with BAP1 mutations. Invest Ophthalmol Vis Sci 2016;27: 2232-2239.

32 Ilagan J, Ramakrishnan A, Hayes B, et al. U2AF1 mutations alter splice site recognition in hematological malignancies. Genome Res 2015;25:14-26.

33 Moore A, Ceraudo E, Sher J, et al. Recurrent activating mutations of G-protein-coupled receptor CYSLTR2 in uveal melanoma. Nat Genet 2016;48:675-680.

Supplementary Information accompanies the paper on Modern Pathology website (http://www.nature.com/ modpathol) 